"On cross and longitudinal sections the periderm is found to consist of one or several layers of primary, and frequently even of secondary bark separated from the deeper living tissues by broad bands of cork. Large groups of sclerenchyma cells with very much thickened porous walls are seen in the periderm and the other parts; their number diminishes toward the interior, and in the inner half of the bast portion they are met with only very sparingly.

"The bast cells are arranged in strictly tangentially elongated groups (as seen on the cross section), but there are no radial rows, therefore the medullary rays are not so readily distinguished with the unaided eye as in some other barks. They invariably make considerable curves where they enter the zones of cribrose and parenchyma tissue, which alternate with those of the bast-fibers; and as they contain a red colored substance they can easily be traced under a low power as undulating red lines. On tangential sections they appear very distinctly as narrow eclipses made up from one to three horizontal, and up to twenty vertical rows of cells. Where these rays pass through parenchyma tissue they are frequently bounded by vertically elongated, rather wide cells, distinguished by the fibrous thickening of their cellulose walls. They form a prosenchyma-like tissue, or in each vertical row of (from five to ten) cells the two terminal ones are pointed.

"The bast-cells are slender and straight, at an average of seventy-five hundredths $\mathrm{mm}$. in length. Each fiber bundle is accompanied by rows of cells with very much thickened lignified walls, each containing a large calcium oxalate crystal (of the monoclinic system) entirely filling the interior of the cell, so that when the crystal is removed by the application of hydrochloric acid, an exact mold of it is left. So closely are these rows of 'crystal sacs' applied to the bast-fibers, that the latter can be brought to view on longitudinal sections only with difficulty. Calcium oxalate crystals of the same form are also seen in other cells, especially in the proximity of the stone cells. Small, rounded, simple starch grains are rather abundant in the parenchyma, and in the cells of the medullary rays can be noticed the red colored masses mentioned above, which are insoluble in alcohol."

Clinical examination of the therapeutic properties of cocillana has engaged the attention of a number of prominent physicians, complete reports of which will be found in various journals. ${ }^{1}$ The latest of these by Dr. John V. Shoemaker, ${ }^{2}$ speaks of his results as follows:

"In small doses, it has a good influence upon the appetite and digestion. In larger quantities, it produces an emetic and cathartic effect. The alvine discharges, under its use contain mucus and bile. It stimulates the sudoriparous glands and, when given in considerable quantities, causes copious perspiration, accompanied by prostration of muscular strength. Excessive amounts also give rise to giddiness. It is eliminated principally by the broncho-pulmonary mucous membrane, upon which it exerts a stimulant and alterative effect.

"Cocillana may be administered in the form of a tincture or fluid extract. The dose of the tincture is from 30 minims to 2 fluidrachms; that of the fluid extract from 10 to 20 minims. In my clinical experiments I have made use of the fluid extract, which resembles sherry wine in color, has an aromatic odor, and a bitter taste. It is, however, not unpalatable when mixed with syrup.

"The chief therapeutic value of cocillana is in the treatment of the diseases of the respiratory tract. In epitomizing my experience with this drug, I deem that I can not do better than to relate briefly the histories of a few cases in which it was employed with notable advantage. Bronchitis is the malady to which it is the most applicable, and it is particularly beneficial in the subacute or chronic stage. Cocillana stimulates the cupillary circulation of the bronchial mucous membrane, and thus relieves inflammatory congestion. The secretions of the respiratory tract become more notable in character; thick and tenacious sputum is liquified, excessive expectoration is restrained, and cough is allayed.

"The amelioration of the cough allows the sufferer to obtain more sleep. The stomach likewise gains in tone under the use of medicinal doses of this drug. I have employed

1 H. H. Rusby, M.D., in the Therareutic Gazette, August, 1888. David D. Stewart II.D, in the Medical News, of August 24,1889 . Reynold W. Wileox, Y.A. M.D., in the New York Medical Journal. Dec. 28, 1889. 1893. it in the bronchitis of children as well as of adults. I have, in some cases of chronic bronchitis, found it fully as efficacious as iodid of potassium, and it is usually better borne, and can be used for a longer period continuously than the iodid. Cocillana should, therefore, be esteemed a very welcome addition to our therapeutic resources. In the doses which I have named, it is totally free from nauseating or depressing effect. In beginning my clinical experiments, I gave the drug alone. When I became acquainted with its properties, I combined it with some syrup for the purpose of increasing its palatability, assisting its action, or fulfilling other indications of the case."

\section{THE SINGLE HYPOPHOSPHITES IN DEFEC. TIVE NUTRITION OF BONE AND NERVE TISSUES.}

\section{BY LEWIS G. PEDIGO, A.M., M.D.} CROCKETT SPRINGS SANITARIUM, VIRGINIA,

Of all the varied uses of the hypophosphites, none are more interesting to me than their employment to meet certain faults of nutrition in the osseous and nervous systems. I group these two departments of the human organism roughly together here, because my attention was directed to them in this connection, by certain rational considerations. In the chemic constitution, the growth and the maintenance of both, phosphorus plays a very important part. So it was inferred, that in certain pathologic conditions of either, especially when characterized by defective nutrition, the use of phosphorus in some convenient and easily assimilable form, is the primary and essential indication of treatment. The various clinical trials of this opinion in practice for several years, have amply justified and sustained the inference. My readers are probably familiar with the use of these remedies in cases of delayed teething in children. Aside from this familiar use, my cases have embraced instances of defective bone development in children, and a certain low type of sub-acute and chronic inflammation, occurring in the bony and periosteal tissues of scrofulous or badly nourished patients, as the result of a blow or injury. It must be borne in mind, in these cases, that the predisposing cause is the state of defective or perverted mutrition. The same traumatism which in such a constitution would cause a troublesome, tedious and prolonged inflammation, would produce very slight local effect in a vigorous healthy subject The course of such a case under the usual treatment is very unsatisfactory. The periostitis shows little tendency to recover, and the bony tissues, so dependent on the healthy action of the periosteum, manifest a tendency to disintegrate. As a result we have a case of hip-joint disease, or Pott's disease, or some other orthopedic affection, with the prospect of a long special treatment, and an endless array of plaster jackets, splints, etc. Recognizing and appreciating all the good to humanity and to science that has been accomplished by the modern orthopedic surgeon, (and it is much), it is plainly the duty and province of the general practitioner, to arrest the progress of these cases in the earlier stage if possible. In my own experience, the hypophosphites offer the best means of relief in this incipient period and, in some cases, almost the only hope of resolution of the inflammatory process. If I might be pardoned the presumption of making a suggestion to a specialist in his own field, I would say to the orthopedic surgeon, that he could not do a hetter thing, even after this ugly inflammation has reached that stage in which 
the tissues break down and deformity results, than put his patient on hypophosphite of lime, as adju. vant to his special surgical measures. Judging by its effects in other fields, and in the first stages of the class of cases under consideration, it would impart more vitality to a failing system, furnish some much needed elements to the organism, and promote healthier action and a tendency to repair in the affected tissues. For obvious reasons, the hypophosphite of lime is the particular salt to be used in all injuries and inflammations of bone and periosteum. With no intention of entering into elaborate review, I recall one case sufficiently typical to exemplify my meaning. A girl 13 years of age was thrown from a horse, falling on the side, so that the left thigh struck the apex of an angular rock. Some lameness resulted -obviously associated with an injury to the femur, near the great trochanter. A slight depression in the bone could be felt at this point. Inflammation, swelling, redness followed, with considerable deepseated tenderness and pain on walking. This condition was temporarily improved by counter-irritation-use of iodids, and various other measures. But there was a constant tendency to relapse, especially in damp depressing weather, with east winds.

The case was becoming chronic, the general health failing, the affected parts threatening a chronic abscess, with more or less disastrous effects upon the integrity of the bone and the future usefulness of the limb. The parents were justly and reasonably alarmed, because one bad case of hip-joint disease had occurred in the family as a result of a similar injury. A scrofulous taint was suspected.

The patient was directed to take a teaspoonful of Gardner's syrup of hypophosphite of lime, three times a day, with no other treatment except rest, suitable diet and hygienic precautions. Improvement began with surprising promptness, considering the peculiar nature of the tissues affected and the usually slow processes which characterize them. The most striking feature was the control of the pain which the remedy seemed to exercise. Of course this was not a direct anodyne influence, but was effected through a modification of the inflammatory processes. The case went on pretty steadily to a good recovery. It is significant that the patient observed the pronounced change in the case as coincident with the change of treatment, and expressed herself as feeling safe so long as she was taking this remedy.

As to the other class of cases alluded to above, I shall present the essential history of only one, which is sufficiently typical of a large number, to answer the purpose of illustration:

A child, male, age 6 months, was suffering with diarrhea and indigestion, associated with difficult and delayed dentition. The gums were red and swollen; teeth were developing slowly which should have appeared about the seventh month. The anterior fontanelle was very large, relatively larger even than it should have been at birth, showing retarded and defective development of the parietal and frontal bones, and indicating a general faulty nutrition, especially of osseous tissue, both in fetal and infantile periods of life. The general appearance of the child was extremely bad. It was ill-nourished, emaciatea, pale, feeble and irritable, with loose, easily wrinkled skin, and a bad complexion. A treatment was adopted for the temporary relief of the dental irritation and diarrhea, and a prolonged course of syrup of hypophosphite of lime was directed, with no other medicinal treatment. I did not see the patient again for six months; (the family lived some distance away). At the end of that time, the mother brought the child to me, not for any professional service (which was obviously not needed) but for exhibition. The improvement was beyond all anticipation. The fontanelle was almost entirely closed; the teething process had gone on without irritation of any consequence and had progressed much more rapidly, and the general condition was so much better, that no one could have identified the child. It was plump, well nourished, of good, clear, rosy complexion, and evidently felt comfortable and in a good humor with the world-a symptom of great significance in these cases.

I have spoken of this case as a typical one-meaning that it represents a class of cases which in my experience have been conspicuously benefited by the use of the hypophosphite of lime. I must say, however, that the brilliant results of treatment in this instance, must not be expected in every case. It is somewhat above the average in this rospect, and therefore not strictly typical in this sense. But its history exemplifies and illustratus principles of treatment that will hold good in all of the class of cases. to which it belongs.

Under the second division of my subject, viz: the use of the hypophosphites as nerve nutrients, it is my purpose to confine myself to one case, a specific: gummatous tumor of the brain:

The patient a bright mulatto, age 26 , stout and generally healthy in appearance, gave a distinct history of syphilis. At the time he came under my observation he was troubled with aphasia, with partial paralysis on the right side, walked imperfectly, scraping the floor lightly with the sole of the right shoe. He could be sent to take a note (not a verbal message), could go to any portion of the town, and find his way without difficulty, but could not remember the names of the streets. He was sent after the cows on the commons, and would get the right ones, but could not tell their names, and if he attempted to throw a stone at one of them, it dropped within two or three paces of his feet. The left hand and arm were much stronger than the right; he was obstinately constipated.

The case was vigorously treated with large doses of iodid of potassium, and small doses of mercury biniodid, with some attention to the digestion and state of the bowels. The iodid was given in increasing quantities until a dose of 60 grains three times a day was reached. This was continued for periods of about a week or ten days, alternating with short periods of suspension of the remedy. The effect was good and improvement began and continued for months. The paresis was better, the mind was clearer and more vigorous, the strength and power of endurance increased and the aphasic symptoms yielded perceptibly. After some months of this treatment, the case seemed to come to a standstill; the improvement reached a limit. Now it was considered safe to begin a restorative treatment-on the theory that the iodid had done its work of liquefaction and absorption of the tumor faithfully and well. The patient was placed on a small dose of tincture of nux vomica, (10 drops) thrice daily before meals and the effect carefully watched. It produced some irritation of the brain-cerebral hyperemia and evident aggravation of all the symptoms. It was withdrawn promptly and the former treatment resumed for another month, with a few doses of bromid of sodium at first, to control the untoward effect of the nux vomica. After a month the nux vomica was again cautiously tried, and this time with good effect. The symptoms-mental and physical-all improved somewhat, and no irritation supervened. After a few days another measure was added to the treatment, viz: the use of a teaspoonful of syrup of hypophosphite of lime, thrice daily, after eating. The effect of this step was perceptible in the decided improvement of all the symptoms. I continued the use of the hypophosphites in this case for several months, watched the effects carefully and with intense interest. I had dispensed from my office only two ounces of the syrup of the lime salt in the first place, so it was soon exhausted. When the patient returned I found I had no more of that preparation, but had a remnant of syrup of hypophosphite of manganese. I substituted this and found, to my surprise, that it seemed to act much more favorably than even the hypophosphite of lime. When this remnant was exhausted, I returned to the use of the lime, however, and the patient noticed the difference in his condition. He insisted that I give him the "same medicine he got the other time," which I did, and with the same result. His neighbors and friends remarked upon the mental improvement. The family reported that he could begin 
to work better, remember messages more accurately, and the patient said he could "learn and recite his Sunday-school lesson better." I had ample opportunity to watch this case closely, and to differentiate the effects of the various remedies used at different times, and my conclusion is that the single hypophosphites were decidedly efficacious in the latter stages of this case.

The theory which led me to their use may be stated as follows :

In cases of gummata of long standing, the contiguous parts of the brain are subjected to constant pressure, interfering seriously, not only with their functional activity, but with the nutrition of the cells and fibers which enter into their structure. After the gummata have been partially or fully absorbed under the influence of the heroic doses of iodid of potassium-so that the brain tissue has the opportunity to expand again to something like its normal size and shape - these compressed, starved and dwarfed fibers and cells need some restorative remedy, some special nerve nutrient, to assist in this development. Nothing so naturally suggests itself to the medical mind for this purpose, as phosphorus in some available form.

As the single hypophosphites are my 'favorite preparation of phosphorus for almost all its uses, I prescribed them in this case, with the results recorded above. I have reason to believe the effects can be duplicated in other similiar cases. In an old case, as this was, even when it first came to me, the physician must not expect too much of any remedy. Parts of the brain structure have been absorbed under the long-continued pressure. Other parts, even though remaining, have been so damaged, that their integrity can not, probably, be fully restored, or their functions rendered strictly normal.

In conclusion, let me warn my reader who feels sufficient interest to test the matter, not to begin the restorative treatment too early in the development of the case.

\section{THE PHYSICIAN AND THE PHARMACIST.}

A Volunteer Paper read in the Section on Materia Medica and Pharmacy at the Forty-fourth Annual Meeting of the American Medical Association.

BY L. CH. BOISLINIERE, M.D. st. Lovis, Mo.

A few words of protest and advice are herein offered from an old practitioner. I believe in the profession of pharmacy and I highly respect the pharmacists. They are the hand-maids of the physicians and our best friends, often our useful advisers. The two professions should work in harmony, one not encroach. ing upon the specialty of the other. Therefore the pharmacist should not tolerate any counter-prescribing, and the physician should not dispense his own medicines. I therefore most strongly protest against the custom, lately too frequently resorted to by physicians, of distributing among their patients any of the potent triturates, or dosimetric granules, so elegantly put upon the market by otherwise reliable wholesale dealers. This practice is enticing, but it is of ten dangerous.

This protest applies chiefly to the city physicians; an exception should be made in favor of the country practitioners. For them it may be sometimes absolutely necessary and is of ten very convenient; they are so placed that they can not do otherwise; but it is a practice fraught with danger.
This practice, in cities especially, may be followed by suits for malpractice in case of any accident. The prescriptions on file with the pharmacist are a strong protection to the physician, as they can be referred to, and, if necessary, brought into court in case of a suit for damages. While serving as Coroner of St. Louis County, several cases of this kind came under $\mathrm{my}$ official notice for investigation. One was the case of a woman, for whom a most reputable physician had prescribed a mild cough mixture. The husband, at the inquest, stated that his wife walked in the garden, after taking a teaspoonful of the medicine, and dropped dead, as if shot. Happily, the bottle of medicine was in the house, properly numbered, and came from a very good pharmacist. The prescription from the file was brought to the house of the deceased and shown to the jury to be a harmless mixture. This, however, did not entirely satisfy the husband. In order to convince him, I opened the deceased's chest and showed him the heart; the pericardium was enormously distended; puncturing it, I received in a cup nearly a pint of serum. The woman had died of acute pericarditis. This amount of serum, pressing on the heart, had caused its sudden stoppage and the patient's death. The husband was satisfied and the physician discharged.

Upon another occasion, an old man had had a weak solution of morphia prescribed for nervousness. The next day I called at the house and found the front parlor full of people and crape at the door. On my inquiring about the patient his sister rushed in, in a fury, and exclaimed to me: "You dare inquire about my brother! You killed him! He walked to the mantelpiece, took a teaspoonful of your infernal mixture and dropped dead!" He died of paralysis of the heart, as was afterwards ascertained. The pharmacist, an excellent one, was requested to come immediately with the prescription, which he had on file, and which called for one grain of sulphate of morphia, of which the patient had taken one teaspoonful, equivalent to one-eighth of a grain, which certainly is not a poisonous dose. The family was finally satisfied that the fatal result was not connected with the medicine.

From these cases is seen the great advantage of filing, dating and numbering prescriptions.

In another instance, there was prescribed for a young woman a one-eighth grain dose of morphia; but, through the accidental misplacement of the bottles, she took one teaspoonful of strong sulphuric acid, and would have died had it not been for copious draughts of water, mixed with plaster scraped from the walls of the room. For several months, she could swallow nothing but milk, as she had an extensive inflammatory stricture of the esophagus.

Here the pharmacist was called in to show that the prescribed mixture was a weak solution of morphia and not sulphuric acid, which it was subsequently learned had been left carelessly in the room to acidulate a battery. I could relate more cases, but these are sufficient to illustrate my position, that of ten the pharmacist is the best protector of the physician.

Another point to be considered is this: Quite a reprehensible custom is that of pharmacists, on demand of patients or their friends, repeating prescriptions without the order of the physician. This is done generally because it would be impolite to refuse; moreover, they have nothing to gain by it. This indefinite refilling of prescriptions should not be 